\title{
San Marcos en 1780
}

\section{DOI DANIEI VALCARCEI}

\author{
Intrcducción.- Antecedentes.- Texto del Informe hecho
} por el Rector Ignacio de Alvarado y Perales

\section{TNTRODUCCION}

El año de 1780 es uno de las más importantes del siglo XVIII en los fastos de nuestra historia. El desasosiego de la época parece culminar, primero, en el conato cusqueño de Lorenzo Farfán de los Godos y el cacique Bernardo Pumayalli Tambahúacso; después, en la más famosa, en la clásica rebelión precursora de una nueva época que se aproximaka: la del ilustre cacique José Gabri=l Tupac Amaru. Por otra parte, extendíase la guerra europea a las colonias de América mediante intermitentes ataques de barcos enemigos, especialmente ingleses, con los consigtientes periuicios al ccmercio espar̃ol. Asimismo la ctisis económica hacía més exigentes a los Oficiales de la Real Hacienda y era iuente de interminables disturbios en los Puebios, Villas y Ciudades de las diferentes Provincias. Poco después se producirá otro sonado y muy peligroso levantamiento, llamado de los Comuneros, en el virreinato de Nueva Granada. Y las nuevas ideas del siglo de la llustración comienzan su obra de dividir a las castas privilegiadias, algunos de cuyos miembros lograrán tomar parte en el proceso de la emancipación peruana.

La real y pontificia Universidad de San Marcos tenía por aquel tiempo como Rector al canónigo doctor Ignacio de Alvarado y Perales (17781783), quien gobernó la Universidad por un lapso mayor al que rezan las Constituciones virreinales, donde se manda que el Rector sea ele- 
gido for un año, pueda ser reelegido por el Claustro durante un período igual, y ratificado por otro año sin elección del Claustro, bastando un simple decreto del Virrey. Alvarado y Perales fué Rector durante cinco años. El motivo de tal extralimitación se encuentra en otro hecho antericr. El virroy Amat promulgó en 1771 un Pegiamento y nombró como Rector al doctor laico Joaquín Bouso Varela (1771-1778). Al concluir su gobierno, y existiendo la tradiciona! àlternativa entre laicos y clérigos, se argumentó que correspondía al nuevo Rector, Alvarado y Perałes, dirigir la Universidad por igual período. Sin embargo - como se verá en parte y ocasión distintas (1) - gracias a la iniciativa del caiedrático de Vísperas de Leyes doctor José Baquíiano y Carrillo, Alvarado y Perales solamente fué Rector durante un quinquenio.

Al comenzar el año de 1780, la Universidad de San Marcos recibió una comunicación del Inspector General de las Armas - don José del Valle y Torres, futuro vencedor del cacique júpac Ainaru- solicitando ayuda económica para los urgentes gastos de la defensa militar, ayuda que fué aprobada en la sesión del día 22 de junio de 1780 . Por entonces la vida de la Universidad pasaba por una etapa caótica. Amat había promulgado un Reglamento - reflejo de actitudes reformistas metropolitanas- que poco después se declaró inoperante. En realidad, prácticamente no se interrumpió la vigencia de las Constituciones de 1735; pero se creó una gran confusión en muchos aspectos del gobierno de la Universidad duranie el tiempo en que-se consideró oficialmente el Reglamento y paralelamente se mantuyo de manera cotidiana aquellas Constituciones, cuya persistencia se explica porque eran suma y compendio de las normas de la Universidad dictados desde el siglo XVI.

\section{ANTECEDENTES}

En 30 de junio de 1780 el virrey Manuel de Guirior envió un decreto a la Universidad, ordenando se normalizase el funcionamiento de las Cátedras y se convocase a Oposiciones, previa publicación de los edictos correspondientes. De esta manera se reanuda los cursos, prácticamente en suspenso por espacio de casi 10 años. El decreto fué leído en la sesión del mismo día 30, fecha anual de la elección del Rector -aunque en las antiguas Constituciones de 1781 se manda realizarla el día primero de julio, víspera de la Visitación de la Virgen. Simultáneamente, por tradicional prerrogativa de la Corona ya mencionada, mandó el Virrey que el reelecto Rector Alvarado y Perales perma- 
neciese al frente del gobierno de la Universidad un tercer período anual sin votáción del Claustro. Es importante recordar cómo, poco después, en la sesión del día 20 de julio, era nombrado catedrático de Vísperas de Leyes el doctor Baquíjano y Carrilo, quien había desempeñado la cátedra de Instituta en la misma Facultad "con la pública satisfacción,.y aplauso que es notorio" (2).

Algún tiempo más tarde, siendo ya virrey don Agustín de Jáuregui, el Rector Alvarado y Perales informó al Claustro, en la sesión del 13 de setiemibre, haber recibido un decreto del Virrey, fechado el día nueve ciel mismo mes, pidiendo a la Universidad informarse acerca del actual estado de los estudios, reglas y métodos en las Cátedras, modo de servirlas por el cuerpo docente, además de todo lo tocante al gobierno de los Colegios y a la enseñarıza de latinidad; y luego, se declarase en suspenso todo lo relativo a las Oposiciones y votación de Cátedras. El doctor Gasnar de Urquisu, Oidor decano de Lima, debía estar presente en las reuniones que celebrase el Claustro para este fin.

El decreto del virrey Jáuregui fué leído por el Secretario Bernabé Cortijo de Vibar. A continuación el Rector agradeció el interés del gobernante por la vida de San Marcos, sobre todo en momentos que graves asuntos de gobierno esperaban preferente resolución. Y estimulado por la actitud del Virrev, prometió presentar su Informe a la brevedad posible, manifestando tener adelantado un trabajo precisamente sobre aquel tópico. La demora en entregarlo cálculaba sería la correspondiente al "tiempo que demandiaba terminar la copia literal de los documentos pertinentes que eran nezesarios para ilustrar su exposición acerca de la Universidad, sus antecedentes y su estado actual. El Rector afirmaba que la mejor prueba del floreciente estado en que tenía la Universidad, lo estaba probando las brillantes actuaciones realizadas por 12 estudiantes del Convictorio de San Carlos en la Universidad, "actuando todos con el mayor primor asi en las lecciones que han hecho de veinte y quatro horas tomando puntos en la letra de Aristóteles como en las Réplicas en que llenos de aquella emulación virtuosa que agita los ingenios, y no deja en las ánimos desabrimiento ni los efectos enconados" (3). Entre los otros elementos que utilizaría para su exposición el Rector Alvarado y Perales, fueron leídas las comunicaciones de 14 de diciembre de 1771 y 31 de marzo de 1772 y el Auto de la Junta de Aplicaciones dado el 10 de abril de 1772 -cuyos textos fueron simplemente mencionados. Concluída la exposición oral del Rector, el Claustro le otorgó, de manera unánime, "todo el poder nesesario" 
para redactar el Informe destinado al Virrey, cuyo texto, fechado el 21 do setiembre e inserto descle fojas 5 vuelta hasta fojas 10 del Libro XIV de Claustros de San Marcos, fué copiacio a continuación de lo correspondiente a la sesión efectuada el día 13 desetiembre de 1780, y es un documento de excepcional importancia para el conosimiento de la historia de San Marcos.

\section{TEXTO DEL INFORME HECHO POR EL RECTOR IGNACIO DE ALVARADO Y PERALES}

INFORME. Ex. ${ }^{\text {no }}$ S.or // En cumplim.to del Sup.or Decreto de VE. de nuebe dal q. corre, sobre q. el R.or y Claustro de esta R.t Univ," le informen el Estado y modo en que se hallan y con q. se efectuan sus estudios el mettodo g. observan los Cathedraticos en el ejersisio de su Enseñanza, los Estaiutos de los Coleg:os en su Doctrina la Regla q. practican los Directores de Latinidad en todas sus clases poniendole a la vista las Constitusiones q se le dieron en su Ereccion, las q. en Junta de aplicaciones le añadio el Ex.mo S.r D. ${ }^{n}$ Manuel de Amat y las ordenes q. posteriormente a estas ultimas se hayan expedido para su govierno, suspendiendose entretanto como se ha observado las votasiones a las Cathejras q. se estan para efectuar: lo q. puedo y debo desir a VE. es que deseando daf prompto obedesim.to a sus Superiores mardatos ordené convocar con el Auto acostumbrado desde el dia anterior para el Miercoles trese del presente anclaustro pleno, haviendo en su Vista concurrido muchos mas de aquellos sugetos de su grernio q. son necesarios para su formasion; y estando sobretodo (sic) presente el S. ${ }^{\text {r }}$ D. ${ }^{\text {ou }}$ Gaspar de Urquisu Ybañes oydor de esta Real Aud." como lo previene VE. lo esté en todas las Juntas q. se hisiesen q. se hubiesen de formar a este numero efecto se paso a conferir tan ymportante asumpto, dandose principio con la letura (sic) publica del espresado Decreto de VE., y finalisada, que fue la materia en cerca de dos horas con toda la quietud, Reverensia, madures q. exigia su deliverasion, y Resividos despues los Votos de los DD. y MM. se determinó por todos el comisionarme, para q. como instruido en todos los puntos q. se exagitaron (sic) hisiese a VE. el informe q. pedia acompañado del testimonio de todos los Documentos q. la Justifiquen: En cuia virtud paso a practicarlo tomando tas cosas desde el veinte y nuebe de Junio de mill sett. ${ }^{\text {ou }}$ setenta $y$ uno q. empeso a tener estta R.' Univ. d una confusion en su Govierno q. hasta el presente no se ha podido desenredar; 
sin duda para q. VE. la saque de este infelise estado en q. yase sumergida, ya q. su mano poderosa al primer paso de su mando la intenta sostener, enmedio de tantas ocupasiones q. es presiso le ocurran, de tanta entidad, importancia, y magnitud.

Hallavase esta R. ${ }^{1}$ Univ.", en aquella posesion de sus Constitusiones $\mathrm{q}$. le formo veinte y dos de Abril de mill quinientos ochenta y uno, en nombre de S. ${ }^{\text {or }}$ D. ${ }^{\mathrm{n}}$ Felipe Segundo $\mathrm{y}$ en virtud de sus R. ${ }^{\mathrm{B}}$ poderes su Virrey el Ex." ${ }^{\text {no }}$ S.or D." Franco. de Toledo por medio de los Visitadores q. nombro para ello D." Diego de Zuñiga Alcalde de esta R. ${ }^{1}$ Aud." y Frey Pedro Gutierres Flores del Orden y Cavalleria de Alcantara sugetos de su Claustro como Doctores q. eran en el, y presediendo el Acuerdo y pareser q. tomó de otros Maestros del propio gremio: Constitusiones q. con los otras q. despues añadieron los Ex. ${ }^{\text {noy }} S .^{\text {res }}$ Marques de Montes Claros en veinte y tres de Agosto de mill seiscientos catorse, y el Principe de Esquilache en nuebe de henero de mil seiscienios dies y nuebe segun las facultades a les habian quedado reservadas por su antesesor confirmó a pedimento del Dor D.11 Ju. de la Reynaga y Salasar del Orden de Santiago y su Proc.ror en Corte la Mag. del S. ${ }^{\text {m }}$ D. ${ }^{n}$ Felipe Quarto en tres de Sep. ${ }^{\text {re }}$ de mill seiscientos veinte y quatro ordenado se cumpla en todo, y por todo el Auto con que se conformó del R. ${ }^{1}$ y Supremo Consejo de las Indias de siete de Junio del mismo (ff.6) año en queßsie havia mandado su cumplimiento observancia $y$ execucion en el entretanto $g$. por el trono no se mandare $y$ ordenare otra cosa, $\sin$ q. los "Virreyes los puedan dispensar, alterar, ni mudar sin Justas y legitimas causas, y dando cuenta de ello al Consejo: se hallaba buelbo a desir en esta posesion esta R.1 Univ. ${ }^{\text {a }}$ quando el veinte y dos del dho. Junio de sett. ${ }^{\circ s}$ setenta y uno, el Ex. ${ }^{\text {no }}$ Sor. D. ${ }^{0}$ Manuel de Amat, empeso a darle otro rostro diverso de aquel q. antes tenia. Proveyo un Superior Decreto poco tiempo antes para q. se hisiese publico en ese dia (q. no lo es segun Ley destinado a la Eleccion annual (sic) de sus Rectores sino el siquiente treinta, mas ocupado en aquel año con la Revista q. pasó del Regimientol de Noblesa de esta Ciudad) nombrando por tal por el tiempo de tres a. al D. or D. ${ }^{\text {on }}$ Joachin Bousso Varela; siendo asi q. ella se devia ejecutar en virtud de sus Estatutos por Votos Secretos de todos sus Doctores y Maestros. Pero ya se espresaba en el q. esto lo practicaba, para q, el fuese quien le pusiese en planta los nuebos Planes q. havia mandado formar a consequensia de los Reales ordenes q. tenia, y q. como primero en esta linea le tocaba haserlo dejando para despues la facultad al Claustro 
aunque mudado el modo Respecto del anterior, segun vimos quando se publicarcn los treinta $y$ tres Articulos que contenian.

Logroso esto en el mes de Sep." del mismo año de mill sett." setenta y uno cerraronse entre tanto y aun despues de ello las puertas de esta R. ${ }^{1}$ Escuela en un todo. L.os sugetos q. habian acabado sus cursos anteriormente pedian el premio de las fatigas en la Borla de D."ir y se les remitia á otro tiempo. Asi corrio hasta el catorse de Diz." del mismo año en q. viendo el activo celo de su Rector el atraso q. resultaba a la Jubentud, y la decadensia $q$. se veia en las Letras no haviendo el incentivo q. se alcansa con la oposision a las Cathedras q. eran a la verdad premio para los Maestros y emulasion para los Escolares, resolvio consultar a su Ex." para q. entretanto q. se ebaquaba el expediente q. ya desde el dose de Octubre havia empesado a seguir sobre nombram." de Concilarios y otros Ministros nuevos para lo q. se requeria conjuntamente con lo q. se debia añadir al sueldo de los Cathedraticos la cont." annual de seis mill quatrocientos sesenta $y$ uno p.", seis y medio R. ${ }^{k}$ sobre los catorce mill novesientos seis $p .^{k}$ y dos Pis. q. actualm. ${ }^{\text {te }}$ gosa en el Ramo de R." novenos se continuase el ejersisio de las Aulas segun las Const."nry antiguas. Remitiose esta a la R.' Junta de aplicaciones donde se havia fundido el nuebo metodo sin q. ninguno del gremio como tal D.r (que eran los q. debian saver mas bien el origen de los abusos q. havia, y el Remedio q. se les debia aplicar) fubiese notisia deel ni menos fuese deputadocara see oydo antes de su formasion, y de donde se esperabase lograseiel Caudah o se nesesitaba aplicandole algunas de las fincas q. no tubiesen especial destino de las muchas q. obtenian los Jesuitas expatriados. Alli con lo q. dijo el S."ir Fiscal de aquella R.' Junta con el primer Auto de ella, de veinte y siete de febrero de setentta y dos con el Sup. ${ }^{\text {r }}$ Decreto q. se intimó al R..r en su virtud, con lo q. nuebamente en treinta y uno de Marzo del mismo año consultó este en su consequensia, y con lo q. por uitimo tubo a bien pedir otra vez el mismo S.nr Ministro Fiscal, remitida q. fue aquella por el Sup. ${ }^{\text {or Gov. }}{ }^{\text {no }}$ a la misma Real Junta pronuncio esta un segundo Auto el dies de Abril, del proprio setenta y dos, en q. ordena y manda q. interin se ebaque el expediente en el todo de sus partes siga el R.or la enseñanza en los terminos antiguos, dando por Vacas las Cathedras q. hubiesen cumplido su tpo., y pasando a poner Edictos a todas ellas para $q$. se lean y obtengan según como se leian, y obtenian antes de q. se hubiese dado metodo diverso para ellas, reservando solo en lo absoluto el dar grados sin espresar de q. en el caso de ser 
presiso dar alguno q. el se debiese practicar en el modo prevenido en los nuebos Estatutos.

Resibido q. fue por el R."r este Auto tan deseado de su actividad pasó a dar por Vacas las Cathedras temporales q. havian cumplido su Trieno ó Quatrienio q. fueron todas entrando aun la de propriedad de Prima de Theologia por haver fallesido antes el D. ${ }^{\text {or }}$ don. Nicolas de Cardenas q. la obtenia. Efectuado asi eligio por Regente de ellas (nombre q. se dá a los q. interinam. te las sirven a mero nombramiento del R. or mientras se proveen por Votos) a los mismos sucuetos q. las havian servido; y en esta ulitma otro D. ${ }^{\text {or }}$ de la Facultad dejandoles solo opcion a todos a la mitad de sus Rentas. Después mando fijar los Edictos de costumbre por el termino ordinario, y aunque se presentaron los q. seguian por entonces esta brillante carrera, no llegó el caso ni de admitirlos a la oposision, ni menos el Acto de los Examenes de las Lecciones Extemporaneas $q$. deben preceder a sus votasiones. Los (f.b.v.) motivos q. le moverian a esto, que sin duda los tendria y quisa reservados, no aparesen ni en el Libro de Claustros ni en alguno otro Documento q. se guarde en el Archivo. Assi no pudiendo desir otra cosa en este punto, solo resta el espresar q. aun en la Conferensia de Grados q. le quedo reservada pidio dispensa para poderlc haser en el modo q. se acostumbraba antes de su ingreso.

Esta consulta q. se dise hecha pocos meses despues q. se proveyó el Auto q. los suspendid GóapareseCen esta Real Escuela para darla en Documento, perōosi ba (sic) el informe q dio en treinta de octubre de sett. ${ }^{\text {"s }}$ seienta y tres pedidole en ocasion de la presentasion q. hiso el Sup." Gob." el el R.P.M. Fr. Man.' Muños del Orden de nro. P." S." Fran." y su actual Prov.' por entonces de su Prov." para q. el Grado Vaco en q. habia sido propuesto por su Difinitorio y votado por el Claustro se le diese segun el estilo antiguo, y no por el moderno, por pedir este cincunstancias q. ya sus años literatura y ocupasiones no le permitian sufrir, quando el otro le era facil en el dia con solo los quinse a. de letura q. havia tenido para obtener la Jubilasion en su Conv. ${ }^{\text {to }}$ fuera de otros muchos q. havia consumido en los principios de sus estudios hasta llegar a ser Lector. En este pues hasiendose cargo de la espresada consulta q. hiso de oficio, y q. no havia tenido proveido la Reproduse dando cinco causales p." a. por termino de ocho a. despues de haviertos los planes modemos se debian dar los Grados como antiguam. ${ }^{\text {te }}$. La resulta q. tubo este expediente fue un Sup. ${ }^{\text {or }}$ Decreto de quinse de Enero de sett. ${ }^{\text {or }}$ setenta y quatro en q. se le debolbia para q. en Claustro determinase segun lo q. tenia propuesto. 
Sin duda q. en el q. se formó para ella, y que no he encontrado se debio deliberar la materia en los mismos terminos q. lo havia pedido pues q. desde entonses no ha havido Grado (exceptando dos q. estos se dieron como lo exigia lo moderno en lo a. mira al acto, pu."o y como lo mondaba lo antiguo en lo $\mathrm{q}$. toza a la leccion secteta $q$. absolutam. " quedaba excluida en aquel metodo. Grados por eso amphibios o de dos raturalesas) q. no se haya conferido conforme a la practica de las primeras confirmadas Constitusiones.

En este estadio nada conveniente al ade!antan. ${ }^{\text {to }}$ de la Jubentud permanecio hasta dies y siete de Julio de sett." setenta y seis q. son los cinco a. q. siguio el Gov."no del Ex. "no Sor. Amat esta $R^{1}$ Univ.". Acavada esta epoca y empesada la de! Ex." S." D." Manuel de Guirior, aun sə mantubo en la misma inaccion por dos años mas en q. no se hiso noveded, consumidos solo por el R..or en reponer a mayor hermosura el Gral destruido antes de su ingreso para bolberlo piesa de la Biblioteca $g$. se le havia aplicado de la Compañia expatriada poniendo en execusion la extinsion q. en el se espresaba del Bibliotecario nombrado, y su expulsion de la Sala q. se llama de las Votasiones y lecoiones secretas donde a consequencia de aquel primer desfiguro havia formado su havitasion perpetua, sin dejarnos donde tenerlas, y cuio defecto se suplia con otra pequeña de clictatura con indesible incomodidad apuntandole coniuntam. ${ }^{\text {to }}$ en virtud del proprio mandato la venia de ochosientos p." q. se le havia aplicadotencsu erecciont a yista de la inutilidad por entonses del tal oficio; y en disponer las cosas nesesarias y oportunas a! recibim. ${ }^{\text {to }}$ de costumbre del nueho $S$. or Virrey cuios montos de las dos partidas sabremos quando presente la cuenta de entrada y salija de su tiempo.

Pasado este espacio de siete.a. en el todo: espacio q. no ha tocado alguno de los antesesores despues q. el Ex. nio S. ${ }^{\text {rr }}$ D. ${ }^{n}$ Fran."o de Toledo en mill quinientos setenta, sacó a la R.' Escuela del poder de la Insigne Religion de S. ${ }^{\text {tn }}$ Domingo donde havia estado desde dose de Mayo ce mill quinientos cincuenta y uno (4) q. fue su primera fundasion, y en cuio tiempo eran sus Priores los Rectores natos transito q. confirmó en treinta de Diz." del siguiente año el S.or D." Felipe Segundo comc q. havia sido por especial encargo y comision de su poder, y en el qual tambien se obtubo a dos de Julio la confirmasion Pontificia del S.or Pio Quinto (5) q. le faltaba: hizo una Reverente Representasion el Cuerpo del Clero a su Ex." pidiedole lo restituiese a la alternativa del Rectorado $q$. debia haver tenido a lo menos quatro a. antes sino se hubiese invertido el orden q. mandan las Const. ${ }^{\text {nex }}$ antiguas no 
derogadas en su primer titulo al Capitulo quinto donde tratan asi espresamente de esta materia, como en lo q. mira al primer año de su Eleccion, al sejundo q. puede haver de reeleccion, y la costumbre de otro tersero, y tal ves un quarto q. dan los Ex. ${ }^{\text {mis }}$ S. ras Virreyes quando les agrada. Esta produjo un Sup.or Decreto de veinte y nuebe de Mayo de mill sett. ${ }^{\text {s }}$ setenta $\mathrm{y}$ ocho por el $\mathrm{q}$. se ordenaba se pasase en treinta de Julio en esta sola ocasion por nesesitar el Rector q. acabaja ese mes mas, para concluir el resivimiento insinuado poco há, a celebrar la (f. 7) eleccion de Rector en alguno de los sugetos del Clero como era uso y costumbre.

Pero antes de q. lisgase el dia prevenido para ello hiso una consulta en veinteytres de Julio el $R$. $^{\text {or }}$ al Superior Govierno inquiriendo si la tal eleccion la deberia haser en el metodo antiguo o moderno, olvidado sin duda de haber solisitado él mismo la dispensia de su observancia, y estar asi mandado tanto tiempo antes. El efecto q. produxo una tan no esperada duda, fue el Decretar el veinte y ocho de Julio el q. todo se ejecutase por la paula del primer estremo segun con toda claridad estaba mandado en el Decreto anterior añadiendo el q. respecto de q. lo mas del Segundo no se havia practicado, y q. lo poco q. se havia hecho por el (cuio obgeto miraba al oidor, grados q. a medias se habian dado y de $q$. se ha hablado arriba) estaba abolida a la solicitud del suplicante, se pásasé duego g. quedase elegido el nuebo Rector a tratar en Claustro pleno todo aquello a. convenia consultar al mayor decoro lustre y adelantam. to de Academia y de sus Estudios teniendo presentes todas las ordenes q. tenia dadas su Mag." sobre este punto tan ymportante y de tanta utilidad a estos sus Dominios.

En su vista llegada q. fue la tarde determinada a tan respetosa (sic) Eleccion caio la suerte por casi todos los votos en mi pequeñes, para un carço q. pide las mayores qualidades de prudensia, actividad $y$ constancia. No obstante considerando q. tantos DD. y MM. pues q. fiaban en mis talentos su govierno debian entreveer lo q. mi moderasion no reconoce aun, acepte el cargo, y me di todo a él, no perdonando fatigas ni penalidades en desempeño de tan honrosa confiansa. Assi a pocos dias q. tomé posesion del oficio cité a Claustro para empesar a dar cumplimiento al Superior Orden q. se produxo antes cuia Alma miraba a q. se formasen otros distintos Planes; de los q. se liamaban modernos impracticables por el defecto de la cantidad de pesos q. havian quedado sin aplicasion, y q. de presente aun no podian tener lugar en los q. se corrigiesen los abusos introducidos en los antiguos con el transcurso de los tiempos viendolo todo con mucha atension y madurés. 
Nada se pudo conciuir de oportuno en el asumpto en esta primera sesion. Solo se ordenó entre tanta variejad de pareseres el q. mə hisiese cargo de solicitar, y y'á en la Secretaria de Camara de SE. ya en el oficio de Govierno, ya en la Oficina de Temporalidades algunos testimonios de Documentos, q. se jusgaban obvios, y presisos para la materia, y q. luego q. los tubiese promptos citase de nuebo a la Junta. Dediqueme a ello con promptitud y eficasia, y haviendo logrado por medio de ella parte de los q. se deseaban se verificó el Segundo Claustro. En este se bino por fin despues de muchos debates de discursos tenidos sin ferbor, y antes con la apasivilidad q. es propria de los savios, a mandar $q$. se hisiese una reforma en el modo de los estudios q. ebitase los Riesgos de esos tales quales abusos q. se notaban en quantos fuese posible con unas Reglas faciles y q. no desdigesen mucho de nuestros antiguos Estatutos. Pero no siendo posible q. alli se formase esto sobre tabla, quando ello pedia por su naturaleza mucha incubasion y travajo, y que quando la multitud de dictamenes podia bolber la materia inespedible para siempre: se añadio diestramente el q. todo se encomendase a quatro sugetos del Gremio, dos Theologos, y dos Juristas q. lo ejecutasen con toda brevedad y'peso. Quedaron estos nombrados en el mismo acto, $y$ fueron aquellos en qrienes se tenia mas confianza segun sus luses, Juisio, y discernimiento q. havian de desempeñar 10 ardua de intento de satisfaccion de la Escuela.

\section{"Jorge Puccinelli Converso"}

Logrose asi por q. en siete de Abril del siguiente año de setenta y nuebe yá se leio todo lo q. habian travajado, en el Clausiro q. cité para este efecto. Paresio conveniente en el despues q. se oyo todo su contenido el darle vista al miembro Proc.or q. tiene por Fundasion la Escuila. Este Respondio en el dose del proprio mes, q. reconosido su Plan con mucho cuidado por su atension lo hallaba exacto en todas sus partes, pidiendo solo una tal qual mudansa sobre la Facultad de Canones, y q. con ella podian presentarsele el Sup."r Gov."10 para que ordenase lo q. fuese de su agrado. Determinose de este modo, $y$ en su complim. to con el Ynforme correspondiente lo puse en quinse de dho. Abril en la Secretaria de Camara de su Ex. ${ }^{n}$ donde hoy deben parar (f. 7 v.).

Despues de haver cumplido con este deber, las ordenes q. fueron viniendo del Ex. ${ }^{\text {mo }}$ S.or Antesesor de VEx. ${ }^{\text {ar }}$ se redusen a pedirme en veinte $y$ cinco de febrero de mill sett. ${ }^{\text {s }}$ setenta $y$ nuebe rason indibidual de las Cathedras q. tenia esta R..$^{1}$ Univ. $^{\mathrm{d}}$ de sus rentas, del Ramo donde se pagaban, de sus fundasiones, de sus confirmasiones, y del 
destino de sus Lecturas, y a dose del mes siguiente puse en poder del Secretario del Virreynato un Informe q. allí estará conprenhensivo y claro de todos estos puntos. En el mismo mandato havia otra segunda parte para q. valiendome de los arbitrios q. me paresiesen oportunos, diese lista de los Maestros de Latinidad que havia en toda' la Ciudad, de su Estado, de su conducta, del estilo de su enseñanza en todas clases, de las calles donde havitaban, y del numero de escolares q. tenian; y con efecto habiendo hecho las pesquisas proprias al descubrimiento de este intento, y haviendolos oydo de palabra, y despues por escrito entregue en quinse del mismo Marzo otro papel separado al mismo Secretario del Virreynato, y q. tambien no faltará de esta ofisina bien prolijo en quanto contiene todo lo q. se deseaba en esta materia. Por este tiempo hise una consulta al Sup. ${ }^{\text {r }}$ Gov. ${ }^{\text {no: }}$ q. no tubo proveido alguno sobre q. si fuese servido me previniese lo resuelto por su superioridad a la consulta verbal q. me significo mi antesesor hiso a su Ex." en asuripto de Biblioteca en su Villete de dos de Diz. ${ }^{e}$ de setenta y ocho en Respuesta del oficio q. en veinte y siete del mes anterior le pasé para saver de ella varios puntos q. nesesitaba para norte de mis designios. Como Yo tenía visto q. a pedimento de veinte y cinco de Enero de sett. ${ }^{08}$ sesenta y ocho del R.or y Claustro e informe del Ex ${ }^{\text {no }}$. S.or Amat q. era Virrey por entonses su Mag. (q. Dios guarde) vino á conceder a esta R. IJniv. segun lo deseaba la Libreria de los Jesuitas Expatriados q. tenian en suicolegio de S." Pablo, y que no hay Don pequeño viniendo de aquella mano poderosa, proyecté, y aun intente con el expediente principiado ( $y$ con lo q. se dira poco despues) el haser una soverbia piesa q. la enserrase, y que asi no andubiese peregrinando de sitio en sitio, sin hallar lugar comodo y desente donde fixar pie a su Deposito. Esta me figuraba Yo q. era la nesesidad mas urgente q. podia tener la Escuela en mi tiempo, porq. veia qu. un vissarro General en todas las partes de su proporsion habia sido destruido para colocarla, y q. la restitusion presíssima de este la ha desterrado al corto ambito, y q. no la presenta de un solo golpe de vista de tres salas de Dictadura, donde no puede tener el brillo q. merese, y cuia partida mantendra siempre aunque se buelba trunca, apolillada y pequeña por venir como esta dicho de la liveral conccesion (sic) de nuestro gran Monarca.

A esto se siguieron quatro Decretos con fecha de treinta de Junio de mill sett.os setenta y nuebe vinieron al Claustro q. estaba Junto p. ${ }^{a}$ la reeleccion q. hiso en $\mathrm{mi}$, usando de sus privilegios y facultades en q. mandaba en el uno su Ex., ${ }^{\text {}}$ se le diese posesion en propiedad de 
la Cathedra de Prima de Theologia r. Figentava el D." Don joh. Ant.

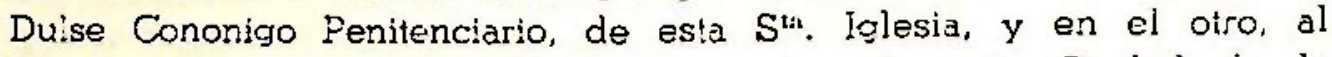
D.or Don Ant. Alvares' Ron Recjidor perpetuo de esta Ciudad die la otra de Prima de Leyes q. oblenia en Regensia en atension a los muchos meritos de ambos a dos, ya que havian estado siete años a media renta en estas y en las otras Cathedras q. havian servido en este tiempo como se executó.

El tersero ordena el precio fixo de milil p." fuera de los Doscientos sesenta y seis p." importe de las Propinas de Examinadores, Ministros, Cajas y Virgen q. deben después de la variedad de Revajas q. ha havido en diversos tiempos tener a los grados mayores q. se empesaron a llamar Yndultos, desde el veinte y nuebe de Mayo de quarenta y tres $\Leftrightarrow$ q. se tubo a bien extinguir en un todo por considerables perjuisios q. resultadan a la Escuela, lo g. entonses se denominaban de Propinas. El fue dimanado de una consulta q. en dies y siete de Junio de setenta y nuebe hise movido de la determinasion q. se tomó para ello en el alaustro q. se celebró en siete del mismo (f. 8) mes a cuios terminos en esta parte vino ajustado el Decreto. Solo se añadio en el q. por lo que miraba a la otra de los segundos Grados de Indulto privilegiados (que tales son aquellos a quienes al principio de esta mutasion se les agració en la rebaja de un tercio en lugar de la mitad de las propinas q. gosaban en los exinguidos quales son los hijos de los Doctores, Maestros, Cathedraticos los g obtayap (sic) Vecas Reales en los dos Colegios extinguidos y refundidos hoy en $S . "$ Carlos, y los q. alcansan Contenta en los casos en q. haviendo fuertes motivos para gastos, grandes e indispensables' urge disminuirlas poniendolas al mismo, ú a otro disiante precio) el $\mathrm{q}$. no solo sean ochosientos $p .{ }^{n}$ los $q$. deban depositar aun que no sea la tersera parte sino ciento treinta y tres $p^{\circ}{ }^{\circ}$ tres rr. mas de la primera porcion como se pedia sino q. en recompensa de esto se les defalcase el tercio menos de los Doscientos setenta y seis p." q. dan todos para las Propinas de costumbre, lo q. no se havia arbitrado, ni propuesto.

El quarto finalm.te tubo por obgeto pedirme puntual rason del sobrante q. debia haber resultado de las Rentas de la Caxa de la R. ${ }^{1}$ Esouela siempre $a$. hallandose vacantes como lo havian estado casi $10-$ das por mucho tiempo, y permanesian aun se Depositan en Regentes a quienes se les asigna unicamente (quando no hay sugetos literatos q. quieran servirla de gracia) la mitad de sus respetivos (sic) honorarios en gratificacion para con su vista $y$ existencia haser saver el destino q. a beneficio de ellas misma, y de uno de los mas illustres 
Cuerpos q. la componen habia dispuesto y meditado su Sup. ${ }^{\text {or }}$ deliberasion. Al punto q. resivi esta apreciable orden Jusgué por presiso y oportuno para ponerlo en ejersisio pasar antes a comunicarle de palabra previamente a su Ex." q. en el año cumplido q. llebaba de Rector, este Ramo se acercaba a quatro mill p. ${ }^{\mathrm{s}}$ y $\mathrm{q}$. aun despues de no haver havido gasto visible que exigiese su espendio no se hallaba en la apariencia integro en la Thesoreria; pero si realm. ${ }^{\text {te }}$ por que en mi poder paraban mill y ochosientos p. ${ }^{8}$ q. habia persebido (sic) de tal año corrido librandolos a mi favor con el titulo de Rector como lo ejecutó mi antesesor en el discurso de siete a. que duró su Rectorado pero espresele al mismo tiempo q. mi designio no habia sido apropiarmelos, ni por un solo momento pues Yo creia firmem. ${ }^{\text {te }}$ y permanesere creyendolo siempre q. aun quando hubiese sido elegido segun lo fue mi antesesor en ese modo q. se denomina nuebo no solo en ese año sino es desde su origen no me podia perteneser en conciencia ese Don, que es el asignado al numero segundo de sus Planes en el punto de los Rectores. Dos causales son las q. expuse a su Ex. ${ }^{a}$ en esta oportunidad me movian a esta accion; la una era el reconoser suplicada a los seis meses la suspension de la execusion de los Planes entre tanto se verificaba el fondo y Ramo de donde el mismo Rector los Cathedraticos, y demas Ministros debian empesar a tener ó acresentar sus Rentas, y haverse en efecto suspendido $\mathrm{p}^{\mathrm{r}}$ el Auto citado antes de dies de Abril de la Junta de aplicaciones, i y turar poreesocaún lo interina sequela de los mencionados antiguos Estatutos; Y la otra era el saver q. la Const. on dies y nuebe del titulo sexto tiene como separados y prevenidos semejantes fondos para gastos y nesesidades para Edifisios, y raparos nesesarios del resinto interno y externo de la $R^{1}$., Escuela, sin que puedan aplicarse a otros diversos destinos, y q. ninguno seria mas distante de esas intensiones que el bolberlos Renta de los Rectores, quienes en verdad hasta setesientos setenta y uno, todos sin excepcion alguna havian servido el empleo por solo el honor q. se desfruta (sic) de el, aun despues de ympender muchos gastos en el tren q. lleba su decoro, y jamas alguno para sacar lucro, y provecho de una camun Madre q. con tanta ternura está antes implorando los socorros y auxilios de unos hijos q. tanto illustra. Descubrile por fin q. mi intento se dirigia a empesar a fabricar con esos pesos, y seguir con los consecutivos de los años venideros una valiente piesa en la misma Escuela correspondiente a su respeto que tubiese lugar de Biblioteca, para colocar los Libros q. estaban encerrados en estantes en tres de las Aulas q. harán falta para las diarias Dictaduras, y Mensales (sic) funciones segun ha- 
blando poco ha de esta materia tengo hecho veer (sic) su urgente necesidad. Aunque a su Ex. a le agradó a primer golpe tan plausible Ydea deiandosele conoser no solo en lo risueño de su semblante sino en lo reiterado de sus espresiones, me previno q. con esa cont. ${ }^{d}$ q. debia quedar separada a su disposision (como asi lo esta aun) era su designio fundar una de la Cathedras mas nesesarias q. tenia pedida la R. ${ }^{1}$ Escuela en sus ultimos Planes, ya por entonses en su poder, y que lo ejecutaria luego q. hubiese caidos Dos mill p. ${ }^{3}$ mas en la The soreria q. compusiesen la cant." de seis mill p. ${ }^{9}$ para hacer la fundasion de Dose mill p. ${ }^{\mathrm{s}}$ (f. 8 v.) teniendo su Ex." los seis mill restantes en arbitrios q. no me comunico, sobre cuio asumpto me repitio varias conferencias esplicandome los vivos deseos q. le asistian de poner en ejecusion esta ymportante obra.

Las dos Cathedras de la Religion de S." Agustin vacas la de Prima del Maestro de las Sentensias (6) y la de Visperas de Dogmas del S. to Doctor se me ordenó por Decretos de dies de Julio de sett."s setenta y dos exhividos despues de las propuestas y sufragios de sus Respectivas fundasiones las pusiese la primera en el R. P. M. fr. Thomas Astuy actual Prov. ${ }^{1}$ de su Provincia, y la segunda en el R. P. M. fr. Joachin Suero Rector del Colegio de S." Yldefonso de esta Ciudad q. observé a la Letra.

Otros Dos Decretos de Veinte de Diz." del mismo año pasado de setenta y nuebe se me remitieron para q. les diese posesion temporal de las Cathedras de Decretocindei Visperassde Canones a los D.res D. ${ }^{\mathrm{n}}$ Domingo Larrion Canonigo de esta S. th $^{\text {ti }}$ Ylesia, y al Marques de Sctoflorido (7) por haver servido varias otras Cathedras por el termino de sus fundasiones Conferidas por el Claustro en Rigorosa Oposision, y en Regensia, en cuia virtud solo habian persivido la mitad del sueldo asignado lo q. se llebó a debido efecto con toda prontitud.

Otra Cathedra de Prima de S.to Thomas propria del Militar Orden de nra. S.ra de las Mercedes vaca por Jubilasion q. obtubo en sus formas el R. P. M. fr. Manuel Concha q. la servia, se mandó por Decreto de veinte y nuebe de Marzo de este año posesionarse en ella como lo ejecuté al R. P. M. fr. Fran.co Ponse q. habia logrado la propuesta en primer lugar de su Difinitorio, el voto de este V. ${ }^{\text {e }}$ Dean y Cav. ${ }^{\text {do }}$ por estar vacante la Dignidad y el del S. or Regente de esta R.' Aud." que es lo que exije su fundasion.

Tres Decretos siguieron a estos; el uno de veinte y ocho de Junio de mill sett. ${ }^{o s}$ ochenta en q. usando su Ex. ${ }^{n}$ de las superioridades -q. le pertenesian me continuaba de R.or por un año mas segun y con- 
forme en vista de ellas lo habian practicado siempre los S.res Virreyes con otros mis antesesores: y los otros dos de treinta del mismo mes y año: El primero de estos dos segundos tiene por objeto la fabrica de un Anfiteatro en el R.' hospital de S." Andres con ynstrumentos proprios y nesesarios para las Dececiones (sic) Anatomicas q. ordena S. $M$. desde el veinte y nuebe de Julio de cinquenta y tres, se ejecuten en el por scndusir al adelantamiento de los profesores; en cuia conformidad aun sin estar arbitrados partes de los fondos de su costo empesé a practicar las diligensias q. se me prevenian para resolber en su ambito el mejor sitio a su formasion, y con el acuerdo del Protomedico Gral del Cathedratico de Anatomia y otros Peritos despues de determinado ya se havian empesado a tirar lineas de su diseño ó Pitipie.

El otro tersero se redujo a q. el dia siguiente pusiese Edictos a la Cathedra de Artes q. desde el año de mill setesientos dies y ocho mando aplicar al R.' Colegio de S. ${ }^{\prime}$ Mrn. el S. ${ }^{\text {or }}$ D. ${ }^{n}$ Felipe Quinto puesto en planta dos años despues, y hoy refundida en el q. se denomina R.' Convictorio de S." Carlos. Assi se ejecutó pasando á admitir a dose de sus s.lumnos q. comparesieron en el espasio de eilos firmardo óposision. Ellos todos leyeron en el termino de veinte y quatro horas resiviendo los puntos en los Libros de Aristoteles segun se practicava desde la mas remota antiguedad, $y$ conforme lo insinuaba el Sup.or Orden. Sus funciones todas fueron mui cump!idas, bien resividas del Auditorio, y algunas de ellas causa de mucha admirasion; pero siempre deiandoseles "conoser $q$. en sus malerias y defensas todo era Repecho por no estar acostumbradas a los principios de este Filosofo sino a los de otros q. se titulaban Modernos. Acavadas estas actuaciones tan gloriosas estaba prevenida su votasion segun siempre se ha acostumbrado para el dia siguiente q. era el Domingo dies del q. corre y en las ultimas horas de sus Visperas, vino el Sup.or Decreto de VE. en q. prohivia todas las q. estubiesen para hacerse en cuia Generalidad quedó esta conprehendida, y así con solo ella mandé se suspendiese del todo. Despues en la misma mañana se me repitio otro (sic) Sup.or Orden q. contenia en particular esta suspension hasiendose con todo cargo de la Universal en virtud de un Recurso q. hiso en su noche el D.or Don Mariano de Orue como uno de los opositores a ellas pidiendome ademas el informe q. debiese haser sobre su intento: el q. en efecto puse el mismo dia en manos de VE. espresando quanto me ocurrio en la brevedad con q. lo tiré para q. no se me notase demora en asumpto de tanta ymportancia, y q. exigia promto Remedio. 
Por ultimo en trese de Julio de este año se Decretó le diese posesion temporal de la Cathedra de Visperas de Leyes con el presiso cargo de acompañar en su enseñansa las del derecho (f. 9) Patrio advirtiendo a sus Discipulos especialm. ${ }^{\text {tc }}$ la disconformidad entre estas y las Romanas conforme al nuebo Plan q. se tenia meditado con arreglo a lo dispuesto en las Universidades e Salamanca y Alcala, al D. ${ }^{\text {or }}$ Don Joseph Baquixano, por estar sirviendo interinam. ${ }^{\text {to }}$ la plasa de Protector Fiscal de Yndios y no tener por tanto tiempo de seguir una oposision Rigorosa, y haver desempeñado la Cathedra de Instituta q. obtenia en Regensia lo q. se efectuo en veinte del mismo mes en q. comparesio a ello, y sobre lo q. ya en el tiempo de VE. se contesta por el D. ${ }^{\text {or }}$ Don Fran.co de Oyague en Autos, q. ha principiado ante mi y paran hoy en recurso ante su gran Justificasion.

Esta historia q. he concluido acortando los hechos quanto he podido para no hacerlo molestosam.te mayorm. ${ }^{\text {te }}$ quando los Documentos en testimonio q. acompaño dan con abundansia las Luces q. se necesitan hasen veer ya la felicidad q. ha tenido esta R.' Escuela de haber podido dar prompto obedesim.to a todos los puntos del Sup.r Decreto de VE. que nos pone en los pensamientos de q. sus principales miras tiran á haserse protector de ella para mejorarla de su antiguo explendor pues con solo su texido se vee q. tiene antes practicado quanto es condusente a su asumpto.

Pero ya es tiempo de desir en breve atVEx: que el estado en que se hallan sus estudios Yyuelcmodoi conngesejefectuan es segun lo previenen las Const. ${ }^{\text {nes }}$ antiguas (cuio exemplar ympreso pongo a su vista en debida forma) (8) por no estar derogadas en nada por S. M. y por estar mandadas obserbar por la Junta de Temporalidades aun sobre aquellos puntos q. tenian oposision con las q. se formaron de orden del Ex. ${ }^{\text {mo }}$ S. ${ }^{\text {or }}$ Amat en aquel mismo lugar.

El tanto ympreso de estas q. paso a manos de VE. equivale al original manuscrito q. se guarda en el Archivo de esta R. ${ }^{1}$ Univ. ${ }^{\text {. }}$. Ellas no han logrado su observancia por falta de la cant. ${ }^{d}$ de seis mill quatrocientos sesenta y un p. ${ }^{\mathrm{B}}$ seis y medio R. ${ }^{\mu}$ q. son nesesarios sobre los q. gosa en el Ramo de Reales novenos, y siempre q. no se verifiquen excequibles, permaneseran impracticables por este capitulo. Este fue el motivo por q. se pidio su dispensa, y se obtubo sin mayor dificultad estendiendose esta despues aun a los Grados de Liz.do y de D.or que se discurrian presisos bajo sus Reglas en caso q. se diesen por la excepsion q. se puso en su concesion quando a $\mathrm{mi}$ veer comprenhendia ambos estremos por lo absoluto de ella. 
Las' Ordenes potseriores q. son las q. dio el Ex. ${ }^{\text {mo }}$ S.or Guirior comprehenden, ya los asumptos de Planes y a lo q. mira a Maestros de Latinidad, y ya lo q. toca a Cathedras. Estos Documentos por lo respectivo a los dos primeros medios, estan ya en la Secretaria del Virreynato, $y$ asi no los exhibo, pero estoy prompto a practicarlo en caso q. se hayan traspapelado y no parescan, no tardando en haserlo, sino el tiempo q. gastasen los Copiantes en trasladarlos del exemplar q. reservé para mi manejo. Mas por lo tocante al ultimo doy testimonio de to dos los Decretos cuios originales estan archivados, añadiendo solo el que en su virtud, hoi solo se cuentan por vacas, y por tanto solo servidas en Regensias q. es lo mismo que tenerlas no por oposision y votacion, sino interinam. ${ }^{\text {te }}$ a nombram. ${ }^{\text {to }}$ del Rector y Claustro hasta q. se provean y q. asi no dan titulo fundado para su opcion: Dos Cathedras de Theologia q. son la de primeras Visperas, y la de Segundas q. por otro nombre se denomina de Nona: dos de artes: dos de Leyes, la de Codigo, y la de Instituta: tres de Medicina, la de Visperas, la de Methodo, y la de Anatomia: una de Lengua Indica, y las tres que eran de los Colegios de S. ${ }^{n}$ Felipe, y $S^{n}$. Martin extinguidos; esto es la de Di gesto Viejo, la del Mro. de las Sentencias, y la de Artes destinadai yá al del Convictorio de S. ${ }^{n}$ Carlos en donde se han refundido aquellos dos primeros.

En Orden al Govierno q. tienen los Colegios q. hoy existen q. son S. ${ }^{n}$ Carlos, y el Seminario de S. to Thorivio Reconvenidos sus Rec tores de su Govierno Jesponden cinen iel año pasado de sett. ${ }^{\text {ss }}$ setenta y nuebe se les pidio por el Sup. ${ }^{\circ}$ Gov. ${ }^{\text {no }}$ rason puntual de sus Estatutos, del numero de sus alumnos y del modo de sus Estudios, y q. haviendo obedesido promptamente lo dieron todo con gran exactitud al Secretario del Virreynato en cuia ofisina pararan, y en el accidente de su perdida estan promptos a exhivir una copia de su contesto. Esto mismo me consta' se les pidio a las Religiones (f. 9 v.) en q. se practican Estudios, y a sus Respectivos Colegios e ygualmente se me ha hecho veer el q. cumplieren con menos escrupulosidad en tan grave asumpto segun lo muestran los exemplares q. exhivieron en la mencionada Seoretaria de Camara donde se les ymponia lo efectuasen en el tiempo limitado que se les señaló.

El destino finalm. ${ }^{\text {te }}$ de los Cathedraticos q. poseen hoy las Cathe dras q. es sobre lo q. unicam.te me resta q. tratar, es ygual al q. siempre ha sido. Pero antes es de notar q. siempre han tenido estós y tie nen dos modos de poseerlas uno de propriedad, y otro por Regensia Los Cathedraticos de Propriedad uno son de propriedad perpetua, y 
otros de propriedad temporal: los Regentes son aquellos en quienes se Depositan las Cathedras interinamente, no por Oposision. Los de propriedad perpetua gosan las Cathedras por el tiempo de su vida, los de propriedad temporal las gosan por el iismpo de su fundasion, $y$ los Regentes por el tiempo de las vacantes ó entre tanto se proveen. Los de propriedad perpetua y temporal gosan la Renta entera, los Regentes solo la mitad a excepcion de si hay quien la sirva de gracia como lo comprehende la Const. ${ }^{\text {on }}$ setenta y una del Ttitulo sexto q. es la Ley treinta $y$ ocho del Titulo segundo de Univesidades, y la Const. ${ }^{n}$ dies y nuebe de dho titulo. Esto supuesto ha sido pues y es hoy el exercisio de unos y otros el de Dictar los Libros sobre q. se leen sus Cathedras q. son para desirlo məjor las asignaturas q. se les tiene destinadas. Asi lo ejecutaban siempre q. havia escolares en quien hacerlo. Ya se vé q. algunas veces en tales quales de ellos ó por sus años, ó por sus accidentes, ó quizá por sus ocupasiones del interés publico no dejarian de haver sus defectos. Pero sea como fuere en los dias de Curso, los mas no faltaban. Tenian oyentes q. escribian ó q. fingian haserlo; Pero como por lo comun estos eran los indibiduos del $\mathrm{R}^{\mathbf{l}}$. Colegio que fue de $\mathrm{S}$." Martin principal porsion de que se componia el Gremio de Estudiantes; esta hoy invertido este orden por el lugar en que se colocó este Colegio. El sitio donde estaba plantado que lo era donde hoy es la Real Aduana tenia oportunamente una gran proporcion al ida y buelta a tarde y mañana de Claustros a Claustros Extinguido este y subrrogado en su lugar el de S." Carlos', ya sus Colegiales no pueden venir diariamente, y aun Jusgo que tienen dispensa superior para no haserlo. Esta retirado dies quadras de esta R. ${ }^{1}$ Escuela; puesto allá en la que era casa de Noviciado de los Jesuitas (9) hasia los arrabales de la portada de Guadalupe. Andarian si viniesen en un solo dia, yendo dos veses y viniendo otras dos quarenta quadras de mal piso. Su vestuario por otra parte no les ayuda para la proptitud. El es en unos de Peluca y Espadin, y en otros de Abates: disconformidad q. no se compone bien con lo uniforme q. debe estar un Congreso Claustral, fuera de los otros distintivos de Vandas en estos, y de charratelas (sic) en aquellos a los hombros para denotar sus diversas graduaciones. Assi nesesitan de Peluquero q. los enpolve y de aseo costoso en todo lo restante del vestido q. no es para un diario continuado. Pero lo mas sensible es, que él yá sollo tiene lugar en esta R..$^{\prime}$ Univ. “ pues con el en ninguna otra parte se les admite. Si se examinan para Curas, si 
se ordenan, si Predican, si Cantan Misa, si leen a las Canongias se desnudan de el, y se ponen havitos clericales. En la R. ${ }^{1}$ Aud. ${ }^{a}$ tampoco los admiten; Y por eso el q. despues de haber finalisado su Jurisprudensia Civil y Canonica quiere practicar las reglas del Foro deja el Vestido, y toma la Golilla, motivo por q. irá perdiendo siempre, y de prompto los indibiduos aprovechados q. le salgan y jamas contará con Maestros enserrados alli por mucho tiempo q. instruian a los otros cursantes, q. principian su carrera: y defecto q. no se esperimentaba con el traje talar de la Opa y Beca, pues q. con el se entraba a los corredores y sus salas como lo hasen hoy los de Santo Thorivio q. lo conservan, y se les admitia como a estos a todos los otros Actos de Altar confeccionario (sic), Pulpito y Banquillo.

Mas dejando esta digresion a q. me ha llevado el Amor de la Patria, y contraiendome de nuebo al mismo asumpto, me parese q. aunque de presente se observe alguna falta en los actuales Cathedratico se verá un equivalente en su manejo de mayor fatiga q. no siendo de su obligasion los pone a cubierto de toda censura. Porq. a la verdad ellos replican, examinan y Presiden en quantos examenes secretos y publicos dan los Carolinos en esta R.' Escuela quando en ella no debian practicarse sino en sus Claustros, como lo hasen los del Seminario (f. 10) y lo parcticaban los de S." Martin por ser funsiones de menor entidad q. no se admiten sino en las Aulas Secretas donde se dicta, y no en su General Polcidestinadonparaslas otras Superiores de Grados de Bachilleres, Licenziados, y Doctores, en los que siempre Presiden y Arguyen, Jamas se escusan en aquellos: siempre los encuentran promptos despues de ser tan repetidos. Solo en los dos años y dos meses q. he corrido de Rector cuento en este Genero dies y nuebe, fuera de los Grados inferiores q. suben a sesenta y ocho y los Superiores a nuebe en $q$. duplican las funciones. Si este no es su deber y con todo lo efectuan, gustosamente porq. la jubentud aproveche, que no harian por dar el lleno a sus obligasiones si hallasen proporcion de oyentes y en su asistencia disposision a la Enseñanza! Ya en los Planes q. estan nuebamente formados, y presentados; se dan arbitrios para remediar este daño, con una durasion y firmesa q. sea invencible en quanto se pueda, viendolos V:Ex." y todo lo demas q. acompaña determinará lo q. fuere de su superior agrado, q. en $\mathrm{mi}$ no hay deseo ni menos pasion, sino solo para q. recibiendo las superiores ordenes de VE." pasarlas a cumplir con toda venerasion y pron- 
titud. Lima y Sep.re, veinte y uno de mill setecientos ochenta $/ D^{\text {ar }}$ Don Joseph Ygnacio Alvarado y Perales //

Es copia de su original.

$\begin{array}{cc}\text { (fdo.) D. Dn. Jph. Yg. Alvarado. } & \text { (fdo.) Eernabe Cortijo de Vibar. } \\ \text { y Perales } & \text { Secret". } \\ \text { (una rúbrica). } & \text { (una rúbrica). }\end{array}$

(1) Historia de la Unlvergidad de San Marcos durante la época de los Borbones (1700-1824) por D. V. (por publicarse) Lima 1951.

(2) Libro XIV de Claustros de la real y pontificia Universidad de San Marcos. Me. Archivo Central "D. Angulo" de la U.N.M. de San Marcos, If. 3v. IVersión literal anotada por D. V. por publicarse).

(3) Ibid if. $4 \mathrm{v}$.

(4) Real Cédula dada en Valladolida por la reina doña Juana y el emperador Carlos V.

(5) Aquí existe un manifiesto errot, (pues la Bula Exponi nobis, dada por Pío $V$ es del dia 25 de jullo de $J 571$ ze ra del díaldos. converso»

(6) Se llamaba así a Pedro Lombardo.

(7) Francisco Antonio Ruiz Cano, Marqués de Sotoflorido, que fué Vice-Rector de San Marcos y no Rector como se ha sostenido.

(8) Alude a las Constituciones de 1735.

(9) Se reliere al actual ediflclo de la Universidad Nacional Mayor de San Marcos, gede del Convictorio de San Carlos desde la expulsión de los Jesuítas hasta la segunda mitad del siglo XViil en cue se trasladó la Universidad de San Marcos, ubicada por entonces en la plaza de la Inçuisición.

v. El actual edificio de la Universidad de San Marcos por Gred Ibscher y Daniel Valcárcel. Imp. Torres Aguirre, Lima, 1951. 